\title{
Prophylactic Zinc Supplementation for Prevention of Acute Respiratory Infections in Infants and Young Children
}

\author{
International Perspective \\ Laura M Lamberti, Christa L Fischer-Walker and Robert E Black \\ From the Department of International Health, Johns Hopkins Bloomberg School of Public Health, Baltimore, MD, USA. \\ rblack1@jhu.edu
}

$\mathrm{A}$ cute respiratory infection (ARI) is a leading cause of morbidity and mortality among young children in low- and middle-income countries. Specifically, pneumonia - an infection of the lower respiratory tract - resulted in 120 million episodes in 2010 and 1.3 million deaths in 2011, among children under five years of age [1]. Approximately $81 \%$ of pneumonia deaths occur during the first two years of life [1], underscoring the need for preventive interventions in infancy and early childhood. Randomized controlled trials (RCTs) have demonstrated the efficacy of prophylactic zinc supplementation on reducing the incidence and severity of acute lower respiratory infections (ALRI) among young children in developing countries $[2,3]$; the majority of evidence, however, has stemmed from studies of routine supplementation with daily zinc doses for an extended period of at least 3 months. Additional research on the efficacy of shortcourse zinc supplementation for $\leq 14$ days is therefore warranted in order to inform the policy-related question of whether interventions of shorter intervals can achieve comparable results to those achieved with routine supplementation [2].

In this issue of Indian Pediatrics, Malik, et al. [4] report the findings of a double-blind RCT assessing the effect of prophylactic oral zinc supplementation for 14 days on the incidence and duration of ALRI among infants 6-11 months of age in an urban area of Delhi, India. This is the first study designed to evaluate the effect of preventive short-course zinc supplementation on the occurrence and severity of subsequent upper and lower respiratory tract infections among infants. Previously published estimates of the effects of short-course zinc supplementation on ARI outcomes have originated from one Bangladeshi study of children aged 12-35 months [5], and three trials assessing ALRI outcomes in the months following zinc treatment for diarrhea among children aged 2-59 months [6-8].

Malik, et al. randomized 272 infants to daily supplementation with placebo or zinc $(20 \mathrm{mg} / 5 \mathrm{~mL}$ elemental zinc as zinc sulfate) syrup for 14 days, and subsequently conducted follow-up visits every two weeks for 5 months to collect morbidity data. Short-course zinc supplementation had no effect on the incidence of all ARI but decreased the incidence of ALRI - defined as report of difficult and/or rapid breathing - by $62 \%$ (95\% CI: $44-$ $74 \%$ ). A previous review of ten randomized trials concluded that preventive zinc supplementation reduced the incidence of childhood ALRI as defined by relatively specific clinical criteria but had no effect if less specific definitions were used [3]. Malik, et al. found the large reduction in ALRI in spite of using a non-specific definition without clinical confirmation. The intervention also decreased the duration of ARI in terms of total number of days of illness during the follow-up period $(15 \% ; 95 \%$ CI: $6-22 \%)$ and number of days per episode $(12 \% ; 95 \%$ CI: $6-22 \%)$.

An important strength of this study was the availability of anthropometric data, which enabled a subgroup analysis confirming similar effects of prophylactic zinc among wasted and stunted infants compared to those of normal weight- and length-for-age. On the other hand, the failure to assess serum zinc levels at baseline was a study limitation indicated by the authors; a sub-analysis comparing effect sizes by serum zinc status would have been a worthwhile addition to existing scientific literature reporting intensified effects of routine and therapeutic zinc supplementation among zinc-deficient children [2]. This study was also limited by a relatively small sample size and the use of caregiver report of signs and symptoms as opposed to a more clinical case definition for diagnosis of ALRI [3]. A larger trial with confirmed clinical outcomes would have been able to determine whether zinc supplementation had an effect on severe lower respiratory infections, including pneumonia, which cause a large proportion of child deaths in India and worldwide [1].

Nonetheless, this study sheds light on the efficacy of 
short-course prophylactic zinc supplementation in reducing the burden of ALRI among infants during the subsequent months. Furthermore, the magnitude and directionality of the effects estimated by this study bolster the conclusions of a previous meta-analysis, which reported no difference between the effects of short-course and routine zinc supplementation trials [9]. Future studies should assess the effectiveness of delivering prophylactic zinc supplementation at-scale, comparing the feasibility and cost-benefit of short-course and continuous regimens.

Funding: None

Competing interests: Robert Black is on the Board of Directors of the Micronutrient Initiative and Vitamin Angels and a member of the Nestle Creating Shared Value Council.

\section{REFERENCES}

1. Fischer Walker CL, Rudan I, Liu L, Nair H, Theodoratou E, Bhutta ZA, et al. Global burden of childhood pneumonia and diarrhoea. Lancet. 2013;381:1405-16.

2. Fischer-Walker C, Lamberti L, Roth D, Black R. Zinc and infectious diseases. In: Rink L (eds). Zinc in Human Health. Amsterdam: IOS Press; 2011. P. 234-53.

3. Roth DE, Richard SA, Black RE. Zinc supplementation for the prevention of acute lower respiratory infection in children in developing countries: Meta-analysis and metaregression of randomized trials. Int $\mathrm{J}$ Epidemiol. 2010;39:795-808.
4. Malik A, Taneja DK, Devasenapathy N, Rajeshwari K. Zinc supplementation for prevention of acute respiratory infections in infants: A randomized controlled trial. Indian Pediatr. 2014;51:780-4.

5. Rahman MM, Vermund SH, Wahed MA, Fuchs GJ, Baqui $\mathrm{AH}$, Alvarez JO. Simultaneous zinc and vitamin A supplementation in Bangladeshi children: Randomised double blind controlled trial. BMJ. 2001;323:314-8.

6. Fischer Walker CL, Black RE. Zinc for the treatment of diarrhoea: Effect on diarrhoea morbidity, mortality and incidence of future episodes. Int J Epidemiol. 2010;39 Suppl 1:i63-9.

7. Baqui AH, Black RE, El Arifeen S, Yunus M, Chakraborty $\mathrm{J}$, Ahmed S, et al. Effect of zinc supplementation started during diarrhoea on morbidity and mortality in Bangladeshi children: Community randomised trial. BMJ. 2002;325:1059.

8. Bhandari N, Mazumder S, Taneja S, Dube B, Agarwal RC, Mahalanabis D, et al. Effectiveness of zinc supplementation plus oral rehydration salts compared with oral rehydration salts alone as a treatment for acute diarrhea in a primary care setting: A cluster randomized trial. Pediatrics. 2008;121:e1279-85.

9. Bhutta ZA, Black RE, Brown KH, Gardner JM, Gore S, Hidayat A, et al. Prevention of diarrhea and pneumonia by zinc supplementation in children in developing countries: Pooled analysis of randomized controlled trials. Zinc Investigators' Collaborative Group. J Pediatr. 1999;135:689-97.

\title{
Zinc for Prevention of Acute Respiratory Infections in Infants - Research Needs
}

\author{
Indian Perspective \\ Archana Patel \\ Program Director, Lata Medical Research Foundation \& Professor of Pediatrics, \\ Indira Gandhi Government Medical College, Nagpur,India.dr_apatel@yahoo.com
}

$\mathrm{P}$ neumonia is responsible for $18 \%$ of under-five mortality as a result of an estimated 151 million new episodes each year occuring mostly in the marginialized and malnourished children in the developing countries who are often zinc-deficient [1]. In fact, the extent of zinc deficiency in a population is often estimated by the prevalence of stunting among these settings [2]. Zinc affects both non-specific and specific immune function at a variety of levels. In terms of nonspecific immunity, zinc affects the integrity of epithelial barrier, and function of neutrophils, natural killer cells, monocytes and macrophages $[3,4]$. Therefore, zinc conceptually promises to offer a beneficial impact on prevention, control and treatment of infections. Based on evidence from several randomized controlled trials and meta-analyses the World Health Organization (WHO) and the United Nations Children's Fund recommended zinc supplementation for up to 2 weeks for management of acute diarrhea [5]. Similarly, several studies evaluated the effect of zinc supplementation in reducing the frequency and severity of respiratory infections children. Variable results have been reported from a series of meta-analyses that evaluated the role of zinc in prevention of pneumonia. Two meta-analyses in 1999 and 2007 reported a beneficial effect in preventing pneumonia in children [6,7]. Subsequently, in 2008, Roth, et al. [8] evaluated the 